\title{
Manometric Tubular Springs Oscillatory Processes Modeling with Consideration of its Viscoelastic Properties
}

\author{
Ismoil Safarov ${ }^{1,2^{*}}$, Muhsin Teshaev ${ }^{2,3,4}$, Sharifboy Axmedov ${ }^{2,4}$, Doniyor Rayimov ${ }^{2}$, Farhod \\ Homidov $^{2}$ \\ ${ }^{1}$ Tashkent Institute of Chemical Technology, Tashkent, Uzbekistan \\ ${ }^{2}$ Bukhara engineering-technological institute, Bukhara, Uzbekistan \\ ${ }^{3}$ Bukhara branch of Institute of Mathematics AS RUz, Bukhara, Uzbekistan \\ ${ }^{4}$ Bukhara branch of the Tashkent Institute of Irrigation and Mechanization Engineering in \\ Agricultural, Bukhara, Uzbekistan
}

\begin{abstract}
This article is dedicated to the operation and management of systems of machine-building and aviation enterprises, systems of production, transport, storage of oil and gas, issues of control of technological processes are of great importance. Control of technological processes is carried out by monitoring the pressure and other parameters. These measuring instruments must have high reliability and the necessary accuracy. In this connection, there is a sharp increase in interest in determining the dynamic parameters of the elements of measuring devices. The main elements of such devices are monomeric tubular springs (Bourdon tubes). The paper considers the natural and forced steady-state oscillations of a thin curved rod interacting with a liquid. Based on the principle of possible displacements, a resolving system of partial differential equations and the corresponding boundary conditions are obtained. The problem is solved numerically by the Godunov orthogonal run method, and the Muller method and the Eigen frequencies found are compared with the experimental results. As a result, for a given axial perturbation, it was possible to select such an effect, in the orthogonal direction, that the amplitude of the longitudinal vibrations of the rod at the first resonance decreased by 20 times. The described vibration damping effect is due to the interrelation of transverse and longitudinal vibrations and is fundamentally impossible in the case of a straight rod.
\end{abstract}

\section{Introduction}

The results of the linear theory of vibrations [1-3] have been used to determine the dynamic properties of pressure gauge tubes. In the future, it is planned to produce high results using simplified formulas of shell theory for the calculation of monomer tubes [4-6].

Much of this scientific paper is devoted to the detection of pipe frequency refraction [79]. The vibrational activity of pressure gauge tubes was studied using the resonance

*Corresponding author: muhsin_5@mail.ru 
separation method [10] used an octagonal cross-section to increase the performance of pressure o tubes. It was proposed to reduce the wall thickness of the pressure gauge on the side parts. Pressure measuring tubes with transverse thickness varying in length were considered in [11]. Under the conditions of pulsation of the working fluid for pressure tubes, vibration effects occur on its elements.

In [12] developed a model with damping devices designed to protect the pressure gauge from pressure pulsation. The effect of the fluid on the vibration damping properties of the pressure gauge is given in [13]. For this purpose, the following liquids are used: water, oil and glycerin. The constructed amplitude-frequency characteristics showed that the most effective liquid is glycerol. The Eigen forms and frequencies of pressure gauge tubes are determined by the theory of vibrations, which are given in $[14,15]$.

In [16], we investigated pressure gauge tubes with variable cross-section for measuring high pressures. The maximum radial stress occurring in the tube is determined. In the present work, the natural and forced steady-state oscillations of the Bourdon tube are considered as a curved rod that oscillates in the plane of curvature (Figure 1). It is assumed that a thin curved rod interacts with a liquid medium. Based on the principle of possible displacements, a resolving system of partial differential equations and the corresponding boundary conditions are obtained.

The solution method is developed, and numerical results are obtained. To compare the results in a simplified formulation, the oscillation equations are obtained from the Dalember principle. The numerical results are compared with the known experimental data.

\section{Materials and Methods}

\subsection{Problem statement and solution methods}

Consider a viscoelastic curved rod (Figure 2) formed by the movement of a flat, possibly variable shape along a flat curve - the center line of a flat curve, so that the center of mass of the forming figure always lies on this curve. The relationship between stresses and deformations is described by Hooke's law with Young's operator modules [17]:

$$
\sigma_{x x}=\tilde{E}_{p} \varepsilon_{x x}
$$

where

$$
\tilde{E}_{\mathrm{p}}[f(t)]=E_{0 \kappa}\left[f(t)-\int_{0}^{t} R_{E \kappa}(t-\tau) f(\tau) d \tau\right]
$$

Where $\sigma_{x x}, \varepsilon_{x x}$ are the components of the stress and strain tensor, respectively; $R_{E \mathrm{~K}}(t-\tau)$ is the relaxation kernel; $E_{0 \kappa}$ is the instantaneous modulus of elasticity, $f(t)$ is an arbitrary function of time. To obtain the equations of motion of a mechanical system, the principle of possible displacements is used; according to this principle, the sum of possible operations of active and passive forces on possible displacements of the system should be equal to zero

$$
\delta A_{n}+\delta A_{x}+\delta A_{p}+\delta A_{t}=0
$$




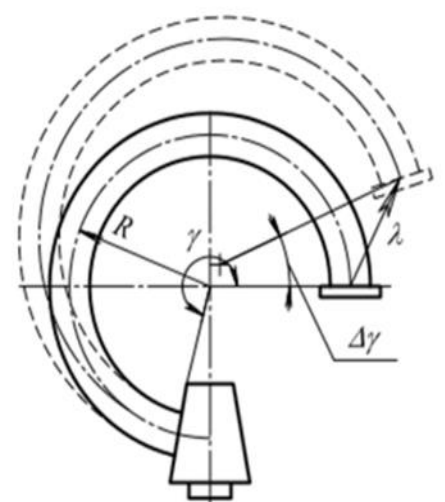

Fig. 1. Pressure gauge spring

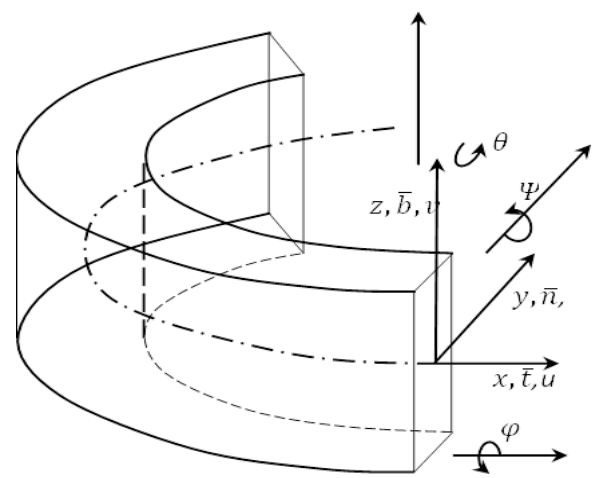

Fig. 2. Element of a curved rod

where, $\delta A_{n}, \delta A_{x}, \delta A_{p}, \delta A_{t}$ are, respectively, the virtual work of normal stresses, torque, inertia forces, and the work of external influences (Figure 2).

The virtual operation of normal voltages can be found from the relations

$$
\delta A_{n}=-\int_{S} \int_{L} \sigma_{x x} \delta \varepsilon_{x x} d x d S=-\int_{L}\left(T \delta \varepsilon+M_{1} \delta x_{1}+M_{2} \delta x_{2}\right) d x
$$

where $\mathrm{S}$ is the area of the normal cross-section; $\mathrm{L}$ is the length of the centerline;

$$
\varepsilon=\frac{\partial u}{\partial x}-K w, \quad x_{1}=\frac{\partial \theta}{\partial x}, \quad x_{2}=\frac{\partial \psi}{\partial x}-K \psi, T=\int_{S} \sigma_{x x} d S, M_{1}=\int_{S} \sigma_{x x} \mathrm{y} d S, M_{2}=\int_{S} z \sigma_{x x} d S
$$

It is not difficult to integrate the relations

$$
T=\tilde{E}_{p} S \varepsilon, M_{1}=\tilde{E}_{p}\left(J_{1} x_{1}+J_{12} x_{2}\right), M_{2}=\tilde{E}_{p}\left(J_{1} x_{1}+J_{21} x_{2}\right)
$$

Moments of inertia are introduced here

$$
J_{1}=\int_{S} y^{2} d S, \quad J_{2}=\int_{S} z^{2} d S, \quad J_{12}=J_{21}=\int_{S} y z d S
$$

where $\mathrm{S}$ is a cross-sectional area. Considering the accepted assumption about the nature of the shear stress distribution, we determine the virtual work of the torque arising in the cross-section.

$$
\begin{gathered}
\delta A_{x}=-\int_{L} M \delta \tau d x \\
\delta A_{p}=-\int_{L} \rho\left\{(\ddot{u} \delta u+\ddot{w} \delta w+\ddot{v} \delta v)+J_{1} \ddot{\theta} \delta \theta+J_{2} \ddot{\psi} \delta \psi+I \ddot{\phi} \delta \phi\right\} d x
\end{gathered}
$$

where $\rho$ is the bulk density of the rod, I is the polar moment of inertia of the normal section relative to the $\mathrm{x}$. Let the vector of external linear forces act along the $\operatorname{rod} \bar{F}=$ $\left(F_{x} F_{y} F_{z}\right)^{\mathrm{T}}$, and at the edge $\mathrm{x}=\mathrm{L}$, the external force $G=\left(G_{x}, G_{y}, G_{z}\right)^{T}$ and the external moment $\left(\mathrm{m}=\left(m_{x}, m_{y}, m_{z}\right)^{T}\right.$ are given) $\mathrm{T}$ is relative to the center of mass of the end of the 
rod. Then the work of external influences on virtual movements is described by the expression

$$
\delta A_{t}=\int\left(F_{x} \delta u+F_{y} \delta w+F_{z} \delta v\right) d x+\left.\left(G_{x} \delta u+G_{y} \delta w+G_{z} \delta v+m_{x} \delta \phi-m_{y} \delta \psi-m_{z} \delta \theta\right)\right|_{x=L}
$$

Substituting in (7) the relations (2), (5) and (6) after the standard integration procedure in parts, we obtain the equations of the law of momentum

$$
\begin{aligned}
& \frac{\partial T}{\partial x}+K Q_{1}-\rho S \frac{\partial^{2} u}{\partial t^{2}}+F_{x}=0 \\
& \frac{\partial Q_{1}}{\partial z}+K T-\rho S \frac{\partial^{2} w}{\partial t}-F_{y}=0 \\
& \frac{\partial Q_{2}}{\partial x}+\rho S \frac{\partial^{2} v}{\partial t^{2}}-F_{z}=0
\end{aligned}
$$

and the equation of the law of conservation of angular momentum

$$
\begin{aligned}
& \frac{\partial M}{\partial x}+K M_{2}-\rho I \frac{\partial^{2} \phi}{\partial t^{2}}+F_{x}=0 \\
& Q_{1}=\frac{\partial M_{1}}{\partial x}-\rho J_{1} \frac{\partial^{2} \theta}{\partial t^{2}} \\
& Q_{2}=\frac{\partial M_{2}}{\partial x}-\rho J^{2} \frac{\partial^{2} \psi}{\partial t^{2}}-K M
\end{aligned}
$$

At the same time, the natural boundary conditions are determined

$$
\begin{array}{lll}
x=0: T=G_{x} & Q_{1}=-G_{y} & Q_{2}=-G_{z} \\
x=L: M=m_{x} & M_{2}=-m_{y} & M_{1}=-m_{z}
\end{array}
$$

The relations (8), (9) and (10) form a closed system of 16 algebraic and differential equations to the vector of unknowns

$$
Y=\left(u, w, v, \varphi, \psi, \theta, \varepsilon, x_{1}, x_{2}, \tau, T, Q_{1}, Q_{2}, M, M_{1}, M_{2}\right)^{T}
$$

If the cross-section of the rod is symmetrical with respect to the normal of the centerline, which is typical for Bourdon tubes, then $J_{12}=J_{21}=0$,

$$
\text { a) } \begin{aligned}
M_{1} & =E_{\mathrm{p} 0} J_{1}\left[x_{1}(t)-\int_{0}^{t} R_{p 0}(t-\tau)_{1} x_{1}(\tau) d \tau\right] \\
T & =E S \varepsilon\left[\varepsilon(t)-\int_{0}^{t} R_{p 0}(t-\tau) \varepsilon(\tau) d \tau\right] .
\end{aligned}
$$




$$
\begin{array}{r}
\text { b) } M_{2}=E_{\mathrm{p} 0} J_{2}\left[x_{2}(t)-\int_{0}^{t} R_{E 0}(t-\tau) x_{2}(\tau) d \tau\right] \\
M=G_{01} S\left[\tau(t)-\int_{0}^{t} R_{G 0}(t-\tau) \tau(\tau) d \tau\right]
\end{array}
$$

It is easy to see that the other unknowns also fall into two groups corresponding to the two subsystems of the equations

$$
\begin{aligned}
& \text { a) } x_{1}=\frac{d \theta}{d x} \\
& \theta=\frac{d w}{d x}+K u \\
& \text { b) } \\
& x_{2}=\frac{\partial \psi}{\partial x}-K \phi \\
& \psi=\frac{\partial v}{\partial x} \\
& \varepsilon=\frac{\partial u}{\partial x}-K w \\
& \tau=\frac{d \phi}{d x}+K \psi \\
& Q_{1}=\frac{\partial M_{1}}{\partial x}-\rho J_{1} \frac{\partial^{2} \theta}{\partial t^{2}} \\
& Q_{2}=\frac{\partial M_{2}}{\partial x}-K M-\rho J_{2} \frac{\partial^{2} \psi}{\partial t^{2}} \\
& \frac{\partial Q_{1}}{\partial x}-K T+\rho S \frac{\partial^{2} w}{\partial t^{2}}-F_{y}=0 \\
& \frac{\partial Q_{2}}{\partial x}+\rho S \frac{\partial^{2} v}{\partial t^{2}}-F_{z}=0 \\
& \frac{\partial T}{\partial x}+K Q_{1}-\rho S \frac{\partial^{2} u}{\partial t^{2}}+F_{x}=0 \\
& \frac{d M}{d x}+K M_{2}-\rho I \frac{\partial^{2} \phi}{\partial t^{2}}-F_{x}=0
\end{aligned}
$$

The system (12a) - (13a) describes a plane bending-longitudinal motion, the system, (12b) - (13b)- orthogonal to it bending-torsional. When calculating thin-walled rods, the first ratio (12a) should be changed to take into account the Pocket effect

$$
M_{1}=E_{\mathrm{p} 0} J_{1} k\left[x(t)-\int_{0}^{t} R_{E}(t-\tau) x(\tau) d \tau\right]
$$

where $\mathrm{k}$ is the coefficient of stiffness reduction due to cross-sectional deformations during bending. This coefficient depends on the shape of the cross-section and the curvature of $\mathrm{K}$. The method of its calculation is given in [17]. The equations of motion of the rod are derived based on the principle of possible displacements. To do this, you need to determine the full virtual work of internal and external forces acting on the rod, including inertia forces. We take the integral terms in (14) to be small, $\varphi(t)=\psi(t) e^{-i \omega_{R} t}$ then the function, where $\psi(t)$ is a slowly changing function of time, $\omega_{R}$ is a real constant. Next, replace the ratio (14) with the following:

$$
\bar{E}_{p}[f(t)]=E_{0}\left[1-\Gamma_{E}^{\mathrm{C}}\left(\omega_{R}\right)-i \Gamma_{E}^{S}\left(\omega_{R}\right)\right][f(t)]
$$

where

$$
\Gamma_{E}^{c}\left(\omega_{R}\right)=\int_{0}^{\infty} R_{E}(\tau) \cos \omega_{R} \tau d \tau ; \quad \Gamma_{E}^{S}\left(\omega_{R}\right)=\int_{0}^{\infty} R_{E}(\tau) \sin \omega_{R} \tau d \tau
$$


The cosine and sine of the Fourier image of the relaxation kernels, respectively; $\omega_{R}$ the real value. The three-parameter Koltunov-Rzhanitsyn relaxation kernel was used in the calculations:

$$
R_{E}(t)=A_{E} e^{-\beta t} / t^{1-\alpha}
$$

\subsection{Natural oscillations of an elastic curved rod}

It is assumed that during the natural vibrations of an elastic rod, all interactions of inelastic origin, including friction, are absent, and the motion obeys the harmonic law

$$
\bar{Y}(x, t)=\bar{Y}_{0}(x) \ell^{i \omega t}
$$

where is the vector of Eigen forms; $\omega$ is the frequency of oscillations. In this case,

$$
\bar{F}=\bar{G}=\bar{m}=0, \beta_{j}=0,(j=1 \ldots 4)
$$

Below are examples of calculating the natural vibrations of an elastic rod, performed following the above theory and the results of experimental studies. Dimensions of the rod (in $\mathrm{mm}$ ): length 210; cross-section 3.1x18.6; radius of curvature 56.8. The small side of the cross-section is parallel to the normal of the centerline. The proper flexural longitudinal vibrations are subject to investigation. Having excluded algebraic connections from equations (12, a), after identical transformations and replacement (13), we have a system of ordinary differential equations resolved to derivatives (here and further, the index for unknown Eigen forms is omitted).

$$
\begin{array}{ll}
\frac{d u}{d x}=\frac{T}{E_{01} \Gamma_{o} S}+K w, & \frac{d w}{d x}=Q_{1}-K u, \\
\frac{d \theta}{d x}=\frac{M_{1}}{E_{01} \Gamma_{o} J_{1} k}, & \frac{d T}{d x}=-K Q_{1}-\rho S \omega^{2} u, \\
\frac{d Q_{1}}{d x}=K T+\rho S \omega^{2} w, & \frac{d M_{2}}{d x}=Q_{1}-\rho S \omega^{2} \theta . \\
\Gamma_{0}=1-\Gamma^{C}\left(\omega_{R}\right)-i \Gamma^{S}\left(\omega_{R}\right)
\end{array}
$$

where for solid rods $\mathrm{K}=1$.

\section{Results and Discussion}

Due to the conditions of fixing on the edges of the rod, the following relations are valid

$$
\begin{gathered}
x=0, u=w=\theta=0 \\
x=L, T=Q_{2}=M_{1}=0
\end{gathered}
$$

It is also necessary to find the co values for which the system of equations with boundary conditions (16) has non-zero solutions. The latter problem was solved 
numerically by the Godunov orthogonal run method, the values found for $\mathrm{E}=19,610 \mathrm{MPa}$, $\rho=8 \mathrm{~g} / \mathrm{cm}^{3}, A=0.048 ; \quad \beta=0.05 ; \quad \alpha=0.1$. The first three natural frequencies of oscillations are shown in Table 1 together with the results of the experiment [18]. According to table 1, the difference between the experimental and calculated data is greater for high frequencies, but in general, it does not exceed $5 \%$.

Table 1. Comparison of theoretical and experimental frequency values

\begin{tabular}{|c|c|c|c|}
\hline Frequency & \multicolumn{3}{|c|}{$\omega / 2 \pi\left(\Gamma_{ц}\right)$} \\
\hline № frequencies & 1 & 2 & 3 \\
\hline Calculation & 75.8 & 212 & 713 \\
\hline Experiment & 75 & 204 & 680 \\
\hline
\end{tabular}

Example 1. Vibrations of a thin-walled curved rod. The proper flexural-longitudinal vibrations of a steel Bourdon tube with a plane - oval cross-section, fixed and rod in Example 1, are investigated. The dimensions of the tube (in $\mathrm{mm}$ ): length 240; wall thickness 0.2 ; large and small semi-axes of the cross-section-9.5 and 3.2; the radius of curvature 45.2. Young's modulus $\mathrm{E}=20.6 \mathrm{x} 104 \mathrm{MPa}$; volume density $\rho=7.8 \mathrm{~g} / \mathrm{cm} 3$; Poisson's ratio $v=0.25$. The formulation of the spectral problem is given by the relations (3), (4). The coefficient of stiffness reduction is 0.129 . The minimum natural frequency obtained when solving the boundary value problem by the orthogonal run method is 88.6 Hz. According to the experimental data, the first resonance of the tube was observed at 100 Hz. The slightly underestimated calculated frequency value is explained by the fact that the rod model does not consider the actual conditions of attachment at the ends of the tube, near which the Pocket effect is less pronounced.

Example 2. Comparative analysis of flexural-longitudinal and flexural-torsional vibrations of a curved rod. The equations of the natural flexural-torsional vibrations of an elastic rod are obtained from the relations $(11),(12, b)$ as a result of the substitution of variables (13). In the form adapted for the implementation of the orthogonal run method, they have the form of a system of first-order differential equations

$$
\begin{array}{ll}
\frac{d \phi}{d x}=\frac{M}{G J}-K \psi & \frac{d v}{d x}=\psi \\
\frac{d \psi}{d x}=\frac{M_{2}}{G J_{2}}-K \phi & \frac{d M}{d x}=-K M_{2}-\rho I \omega^{2} \phi \\
\frac{d Q_{2}}{d x}=\rho S \omega^{2} w & \frac{d M_{2}}{d x}=Q_{2}+K M_{2}+\rho J_{2} \omega^{2} \psi
\end{array}
$$

A rigidly pinched rod with a free end corresponds to the boundary conditions

$$
\begin{array}{r}
\mathrm{x}=0, \phi=\psi=\vartheta=0 ; \\
\mathrm{x}=\mathrm{L}, M=Q_{2}=M_{2}=0
\end{array}
$$

Table 2 shows the calculated and experimental values of the first two natural frequencies of flexural-torsional vibrations of a straight and two circular pipelines with a fixed liquid fixed in supports. The tests were carried out on a stand [19] designed to study the parametric vibrations of elastic pipelines of aircraft structures [19]. The pipe samples are made of $12 \times 18 \mathrm{H} 1$ OT steel with an outer diameter of $8 \mathrm{~mm}$ and an inner diameter of 7 $\mathrm{mm}$. 
Table 2. Frequency change depending on the angle and length of the rod

\begin{tabular}{|c|c|c|c|c|c|}
\hline \multirow{2}{*}{ Solution angle } & Length & \multicolumn{2}{|c|}{ 1 frequency $(\mathrm{Hz})$} & \multicolumn{2}{c|}{2 frequency $(\mathrm{Hz})$} \\
\cline { 2 - 6 } & $(\mathrm{mm})$ & calculation & exp. & Calculation & exp. \\
\hline $0^{\circ}$ & 800 & 64.2 & 61.2 & 177 & 166 \\
\hline $90^{\circ}$ & 765 & 64.7 & 64.1 & 185 & 170 \\
\hline $180^{\circ}$ & 625 & 84.0 & 61.5 & 243 & 170 \\
\hline
\end{tabular}

The density of the pipe material is $7.9 \mathrm{gm} / \mathrm{cm}^{3}$, and the elastic modulus is $\mathrm{E}=18.0$ * $104 \mathrm{MPa}$ [4], the Poisson's ratio is $v=0.3$. The working fluid used was AMG-10 oil with a density of $0.85 \mathrm{~g} / \mathrm{cm}^{3}$ (GOST 6794 - 75). In this case, the reduced density of the tube, taking into account the mass of the liquid, is $9.5 \mathrm{~g} / \mathrm{cm} 3$. According to Table 3, the calculated data are better consistent with the experience with a lower curvature of the tube. Still, in the latter case, concerning the work [19], the possibility of loosening the attachment is not excluded. Solutions to boundary value problems (22), (23), (24), (25) we studied the parameter of the curvature of the axis on the example of the vibrations of the rod with the following dimensionless geometric relations numerically

$$
\frac{S L}{K J_{1}}=7238 ; \frac{S L}{J_{2}}=1505 ; \frac{S L^{2}}{I}=1246 ; \frac{E J_{2}^{2}}{G L}=3.865
$$

For each type of vibration: flexural-longitudinal and flexural-torsional, Tables 3 and 4, respectively, show the values of the first five natural frequencies of co, depending on the value KL, which characterizes the degree of curvature of the centerline. The calculation was carried out without taking into account the inertia of the rotation of the cross-section. The unit of time is considered equal to $\mathrm{L}(\mathrm{p} / \mathrm{E}) 1 / 2$.

Table 3. Frequency change depending on the parameter that characterizes the degree of curvature of the centerline (flexural-longitudinal vibrations)

\begin{tabular}{|c|c|c|c|c|c|c|}
\hline \multirow{2}{*}{$\mathrm{N}$} & \multirow{2}{*}{$\mathrm{KL}$} & \multicolumn{5}{|c|}{ Eigenvalues } \\
\cline { 3 - 7 } & & 1 & 2 & 3 & 4 & 5 \\
\hline 1 & 0 & 0.04133 & 0.2590 & 0.7252 & 1.421 & 1.571 \\
\hline 2 & 0.78 & 0.04184 & 0.2425 & 0.7043 & 1.383 & 1.737 \\
\hline 3 & 1.57 & 0.04345 & 0.2091 & 0.6582 & 1.334 & 2.044 \\
\hline 4 & 3.14 & 0.05046 & 0.1593 & 0.5433 & 1.207 & 2.090 \\
\hline 5 & 4.71 & 0.06453 & 0.1413 & 0.4240 & 1.043 & 1.910 \\
\hline 6 & 6.28 & 0.08920 & 0.1463 & 0.3237 & 1.8656 & 1.684 \\
\hline
\end{tabular}

Example 3. Vibrations of a rod with a load at the end. Let one end of the rod still be sealed, and at the free end a load is rigidly fixed, which we will consider non-deformable. We show how the inertia of the load is taken into account in the boundary conditions. The kinetic energy of a solid body, according to [20], is determined by the expression

$$
T=\frac{1}{2} \int(\bar{V}+\bar{\Omega} \bar{r})^{2} \rho \bar{r} d U
$$


where the integration is performed over the entire volume of the body $U$ of the density $p_{r}$; $\Omega$ is the angular velocity; $\mathrm{V}, \mathrm{r}$ are, respectively, the velocity of an arbitrary reference point rigidly connected to the body and the radius is the vector from this point to the center of mass - opening the brackets in (7) and performing the integration.

Table 4. Frequency change depending on the parameter that characterizes the degree of curvature of the centerline (flexural-torsional vibrations)

\begin{tabular}{|c|c|c|c|c|c|c|}
\hline \multirow{2}{*}{$\mathrm{N}$} & \multirow{2}{*}{ KL } & \multicolumn{5}{|c|}{ Eigenvalues } \\
\cline { 3 - 7 } & & 1 & 2 & 3 & 4 & 5 \\
\hline 1 & 0 & 0.09063 & 0.5680 & 1.590 & 3.117 & 5.153 \\
\hline 2 & 0.78 & 0.07472 & 0.3827 & 1.319 & 2.822 & 4.843 \\
\hline 3 & 1.57 & 0.06633 & 0.2809 & 0.9891 & 2.362 & 4.305 \\
\hline 4 & 3.14 & 0.07672 & 0.2240 & 0.7271 & 1.896 & 3.680 \\
\hline 5 & 4.71 & 0.1045 & 0.2026 & 0.5272 & 1.485 & 3.066 \\
\hline
\end{tabular}

$$
T=\frac{1}{2} \mu \bar{V}^{2}+\mu \bar{r}_{0} \overline{V \Omega}+\frac{1}{2} \bar{\Omega} J \bar{\Omega}
$$

where $\mu=\int \rho_{r} d U$ is cargo weight; $\bar{r}_{0}=\frac{1}{\mu} \int \bar{r} p_{r} d U$ is radius is the vector from the reference point to the center of mass; $\mathrm{J}$ is the inertia tensor of the load at the reference point. As a reference point, we take the point of the centerline $\mathrm{x}=\mathrm{L}$. Then, taking into account the conditions for securing the load, as well as the notation of the positive turns of the section in Fig. 1, we can write the coordinates of the vectors $\mathrm{V}$ and $\Omega$ in the basis $(\bar{t}, \bar{n}, \bar{b})$ :

$$
X=L ; \quad \bar{V}=\frac{d}{d t}(u, w, v)^{T} ; \bar{\Omega}=\frac{d}{d t}(\varphi-\psi, \theta)^{T}
$$

The components of the vector $r_{c}$ and the tensor $\mathrm{J}$ in the same coordinate system are denoted as follows

$$
\bar{r}_{c}=\left(x_{c}, y_{c}, z_{c}\right)^{T} ; J=\left\{J_{i j}\right\},(i, j=x, y, z)
$$

Taking into account (20), (21), the coordinate representation of the kinetic energy (19) has

the form

$$
T=\mu\left(\frac{1}{2}\left(u^{2}+w^{2}+v^{2}\right)+y_{0}(\dot{v} \dot{\phi}-\dot{u} \dot{\theta})+x_{0}(\dot{w} \dot{\theta}-\dot{v} \dot{\psi})+\right.
$$

$$
+\frac{1}{2}\left(J_{y y} \dot{\psi}^{2}+J_{z z} \dot{\theta}^{2}+J_{x y} \dot{\phi}^{2}-J_{y z} \dot{\psi} \dot{\theta}-J_{y z} \dot{\psi} \dot{\phi}+J_{y z} \dot{\theta} \dot{\phi}\right)
$$

Hence, ignoring the time variation of the vector $\bar{r}_{0}$ and tensor at small motion amplitudes and calculating the virtual work of the inertia forces acting on the load in a linear approximation using the D'Alember principle, we obtain the natural conditions of the rod oscillation at the edge $\mathrm{x}=\mathrm{L}$ : 


$$
\begin{aligned}
& Q_{1}=\mu\left(\ddot{W}+x_{0} \dot{\theta}-z_{0} \ddot{\varphi}\right), \\
& Q_{1}=\mu\left(\ddot{v}+x_{0} \ddot{\psi}-z_{0} \ddot{\varphi}\right) \\
& T=\mu\left(-\ddot{u}+z_{0} \ddot{\psi}-y_{0} \ddot{\theta}\right) \\
& M_{1}=-\mu\left(x_{0} \ddot{W}+y \ddot{u}\right)-J_{z z} \ddot{\theta}-J_{z x} \ddot{\varphi}+J_{z y} \ddot{\psi} \\
& M_{2}=-\mu\left(x_{0} \ddot{v}-z_{0} \ddot{W}\right)-J_{z z} \ddot{\theta}-J_{z x} \ddot{\varphi}+J_{z y} \ddot{\psi}
\end{aligned}
$$

From the conditions (22), it follows that the center of mass of the load does not lie in the plane of the centerline or the centrifugal moments of inertia $J_{z x} . J_{z y}$ are different from zero, then the flexural-torsional and flexural-longitudinal vibrations are related to each other even if the corresponding unknowns in equations (12), (13) are separated. Consider a rod with the parameters specified in Example $3(\mathrm{KL}=3.14)$ and assume that the load is fixed symmetrically relative to the plane of the centerline. Then $z_{0}=J_{z x}=J_{z y}=0$, and the flexural-longitudinal vibrations do not depend on the flexural - torsional ones. In the case of natural oscillations. the conditions (17) after replacing (1) are converted to the form

$$
\begin{aligned}
T & =\mu \omega^{2}\left(u+y_{0} \theta\right), \\
Q_{1} & =\mu \omega^{2}\left(-W-x_{0} \theta\right), \\
M_{1} & =\mu \omega^{2}\left(x_{0} W-y_{0} u\right)+J_{z z} \omega^{2} \theta, \\
M & =\omega^{2}\left(\mu y_{0} v+J_{x x} \phi+J_{x y} \psi\right), \\
Q_{2} & =\mu \omega^{2}\left(-v-x_{0} \psi-y_{0} \phi\right) \\
M_{2} & =\omega^{2}\left(\mu x_{0} v+J_{y y} \psi-J_{y z} \phi\right)
\end{aligned}
$$

Let the load be made in the form of a rigid homogeneous beam with a length of $0.3 \mathrm{~L}$. and $\mathrm{y}_{0}=0$. For the above parameters of the rod and load in Table 5, the first five eigenvalues of the boundary value problem are given (23). (24), (30), found by the orthogonal run method, depending on the weight of the load (Table 5).

Table 5. Natural frequencies of the rod as a function of body weight

\begin{tabular}{|c|c|c|c|c|c|}
\hline $\mathrm{M}$ & \multicolumn{5}{|c|}{ Eigenvalues } \\
\hline 0 & 0.05044 & 0.1591 & 0.5419 & 1.198 & 2.064 \\
0.1 & 0.04207 & 0.1161 & 0.3500 & 0.8259 & 1.499 \\
0.5 & 0.02770 & 0.07410 & 0.2838 & 0.7400 & 1.386 \\
1 & 0.02110 & 0.05784 & 0.2612 & 0.6969 & 1.312 \\
\hline
\end{tabular}

For thin curved tubes, it is found that the radius of curvature decreases, the real part of the natural frequency decreases, and the attenuation decrement also decreases markedly.

The obtained numerical results were compared with the numerical results obtained from the analytical solution $[21,22]$ for the same values of parameters. The difference in results was up to $12 \%$.

The research results can also be used in the development of a new design for drying cotton seeds $[23,24]$, as well as in improving the energy efficiency and reliability of the power supply $[25,26]$.

Here it was also possible to consider the control of body vibrations by introducing servo constraints [27-29], which will be considered later. 


\section{Conclusions}

1. With an increase in the frequency of natural oscillations, the attenuation decrement increases in the presence of internal friction and decreases in the presence of external friction. Moreover, with an increase in the intense dissipation, aperiodic modes (purely imaginary eigenvalues) arise, starting from the highest numbers of Eigen movements, in the case of internal friction. and from the lowest numbers of Eigen movements, in the case of external friction.

2. In the case of homogeneous dissipation, the Eigen forms coincide with the corresponding Eigen forms of elastic vibrations. In the case of internal friction for vibrational modes, the imaginary parts of the eigenvalues depend linearly on the parameter. In the case of external friction, the imaginary part of the different eigenvalues is the same, does not depend on the curvature of the axis, and is determined by the expression:

$$
\operatorname{Im} \omega=\frac{J_{1} k}{2 S L^{2}} \stackrel{\sim}{a 2}
$$

3. If the friction is inhomogeneous, the eigenforms depend on the intensity of the dissipation, and the oscillations of the various spatial points are phase-shifted. The viscoelastic rod model makes it possible to study forced steady-state oscillations at resonances by taking into account the energy dissipation.

\section{References}

1. Korn G and Korn T, Handbook of Mathematics (Moscow: Nauka), (1978)

2. Lurie A I, On small deformations of curved rods (Moscow: Nauka), (1966)

3. Novozhilov V V, Theory of thin shells (L Sudpromgiz)(1962).

4. Svetlitsky V A,Mechanics of flexible rods and threads (Moscow: Mashinostroenie). (1978)

5. Chuba A Yu. Pirogov S P and Smolin N, And Determination of natural vibration frequencies of curved pipes of non-circular cross-section Proceedings of Higher Educational Institutions, Oil and Gas, Tyumen No 1 pp 77-82

6. Chuba A Yu. Pirogov S P and Dorofeev S M, Definition of proper frequencies of oscillations of manometric tubular springs Izvestiya vysshikh uchebnykh zavedeniy "Neft i gaz" Tyumen No 2 pp 70-74. (2007)

7. Panovko Ya G, Fundamentals of the applied theory of vibrations and shock (Moscow: Librocom) p 274 (2010)

8. Skhirtladze A G Bykov S Yu and Skhirtladze S A, Restoration of operability of spring devices for pressure measurement Izvestiya Volgogradskogo gosudarstvennogo tekhnicheskogo universiteta ,7 pp 50-54. (2013).

9. Timoshenko S, Fluctuations in engineering (Moscow: KomKniga). (2006).

10. Chekhov V I Kalinichenko A N, Research of ways to reduce the influence of vibration on manometric equipment Bulletin of Siberian Science 3 pp 60-64. (2013)

11. Cherentsov D.A., Determination of generalized resistance forces in the Lagrange equations of the second kind. describing the oscillations of a manometric spring in the presence of viscous friction forces Natural and technical sciences, 4 (72) pp 12-15

12. Cherentsov D A Pirogov S P, Study of damped oscillations manometric tubular spring in a viscous medium News of higher educational institutions. Oil and Gas 5 pp 66-72. (2014) 
13. Champain L.F., Gladwell I., and Thompson S., Solution of ordinary differential equations using the MATLAB package version, textbook (translated from the English by Makarov I.A., Saint Petersburg [et al.]: Lan) p. 299, (2009)

14. Rossikhin Yu A Shitikova M V and Ovsyannikova E I, Analysis of fractional derivatives of free damped oscillations of a circular cylindrical shell Proceedings of the 5th International Conference on Vibration Problems, OVP-2001 8-10 October 2001 Moscow M: IMASH pp. 378-381, (2002)

15. In Il Moiseev A, Boundary control of string vibrations minimizing the power integral $\mathrm{p}>1$ of the control module or its derivative remote control, (68), 2 pp 313-319, (2007).

16. Changyu Z. Flourish. Ming $\mathrm{X} \mathrm{Z}$ and Zhongqin L, A strategy for suppressing parametrically excited lateral oscillations of a mass-loaded string J. Southeastern University 20 (2) pp 165-169, (2004)

17. Mirsaidov M. Safarov I and Teshaev M, Dynamic instability of vibrations of thinwalled composite curved viscoelastic pipes under the action of pulsed pressure, E3S Web of Conferences 164 14013, (2020)

18. Kaplunov Y.D., and Wilde M.V., Boundary and interphase oscillations in elastic shells of rotation Mathematics. Phys (DEPUTY), 51 pp 29-48. (2000)

19. Li Shi-rong 2000 Gansu the Gong dasya of cubao j. Gansu Univ. Technol 26 N 2 pp 98-102

20. Magnitsky K and Shits V, The stability of ellipsoidal heads cylindrical high-pressure vessels Apple. Mech. and eng, 5 (2) pp 389-404, (2020)

21. Durdiev D K and Totieva Z D, The problem of determining the one-dimensional kernel of viscoelasticity equation with a source of explosive type Journal of Inverse and Ill-Posed Problems 28 (1) pp. 43-52, (2018)

22. Durdiev D K and Rahmonov A A, Inverse Problem for A System of IntegroDifferential Equations for SH Waves in A Visco-Elastic Porous Medium, Global Solvability Theoretical and Mathematical Physics (Russian Federation) 195(3), pp 923-937 (2018)

23. Rahmonov X K, Improvement of Equipment and Technology of Drying of the Cotton Mass and its Technological Assessment on the Basis of its Thermal Properties International Journal of Advanced Research in Science. Engineering and Technology 65 May (www.ijarset.com) (2019)

24. Rahmonov X K, Development of a New Design for Drying Cotton Seeds with Purpose of Efficient Use of Heat International Journal of Advanced Research in Science. Engineering and Technology 74 May (www.ijarset.com), (2020)

25. Sadullayev N.N. Safarov A.B. Nematov S N and et al, Opportunities and Prospects for the Using Renewable Energy Sources in Bukhara Region Appl, Sol Energy 56 pp 291-300 https://doi.org/10.3103/S0003701X20040106, (2020)

26. Sadullaev N N. Mukhamedkhanov U.T. Nematov SH N and Sayliev F O, Increasing Energy Efficiency and Reliability of Electric Supply of Low Power Consumers, International Journal of Engineering Trends and Technology 68(12) pp 43-47 doi:10.14445/22315381/IJETT-V68I12P208. (2019)

27. Khusanov K, Stabilization of mechanical system with holonomic servoconstraints. IOP Conf Se:r Mater Sci Eng 883, 012146 (2020)

28. Khusanov K, Stabilization of mechanical system with nonholonomic servoconstraints constraints. IOP Conf Ser: Mater Sci Eng 883, 012164. (2020)

29. Khusanov K, Equations of motion of mechanical systems with nonlinear nonholonomic servoconstraints. IOP Conf Ser: Mater Sci Eng. 869, 072021, (2020) 